\title{
Editorial
}

\section{Wound Practice and Research: staying connected to solve global challenges}

\section{Cowin A and Wilson B}

For referencing Cowin A and Wilson B. Globally important, nationally relevant: Wound Practice and Research in 2020. Wound Practice and Research 2020; 28(1):4.

DOI https://doi.org/10.33235/wpr.28.1.4

So far, 2020 has been a challenging year for many with unprecedented bushfires, storms affecting large parts of Australia and now the global impact of the COVID-19 pandemic. No one will come out of 2020 unaffected by the events that are unfolding around the globe and many will have experienced first-hand these effects on families, homes and workplaces.

Just a few short weeks ago, many of us were looking forward to joining the largest gathering of wound care professionals in the world, at the World Union of Wound Healing Societies in Abu Dhabi. Our excitement at learning about new wound research and management alongside catching up with our global colleagues and updating our professional development was put aside to deal with more pressing healthcare challenges. As we move forward, maintaining connections with our international colleagues will be an increasingly important and an ongoing issue as we deal with the current and future implications of COVID-19.

Prof Allison Cowin

Editor Wound Practice and Research

Blake Wilson

CEO Wounds Australia
Sharing information and communicating our research through Wound Practice and Research is one way that Wounds Australia is keen to facilitate new global initiatives and help maintain our international focus. As the world continues to move to online platforms, it is timely that we can announce the launch of our new digital version of the journal which will increase access to Wound Practice and Research to many new readers, will improve our international profile and will help achieve Wounds Australia's objective to reduce the harm caused by wounds. Be assured that we will continue to produce our hard-copy journal for all our Wounds Australia members, we recognise its importance for our community and have put a lot of thought into refreshing the look of the journal - we hope you approve.

In these times of uncertainty, Wound Practice and Research will continue to be the rigorous and consistent source of relevant information you have come to expect. Through the new online version, we hope to extend the benefits even wider to our membership and beyond. We know that it is now more important than ever that society broadly, and the medical community specifically, works together for the common good. Together we can all build a kinder and more supportive society.

Wound Practice and Research online edition woundpracticeandresearch.com

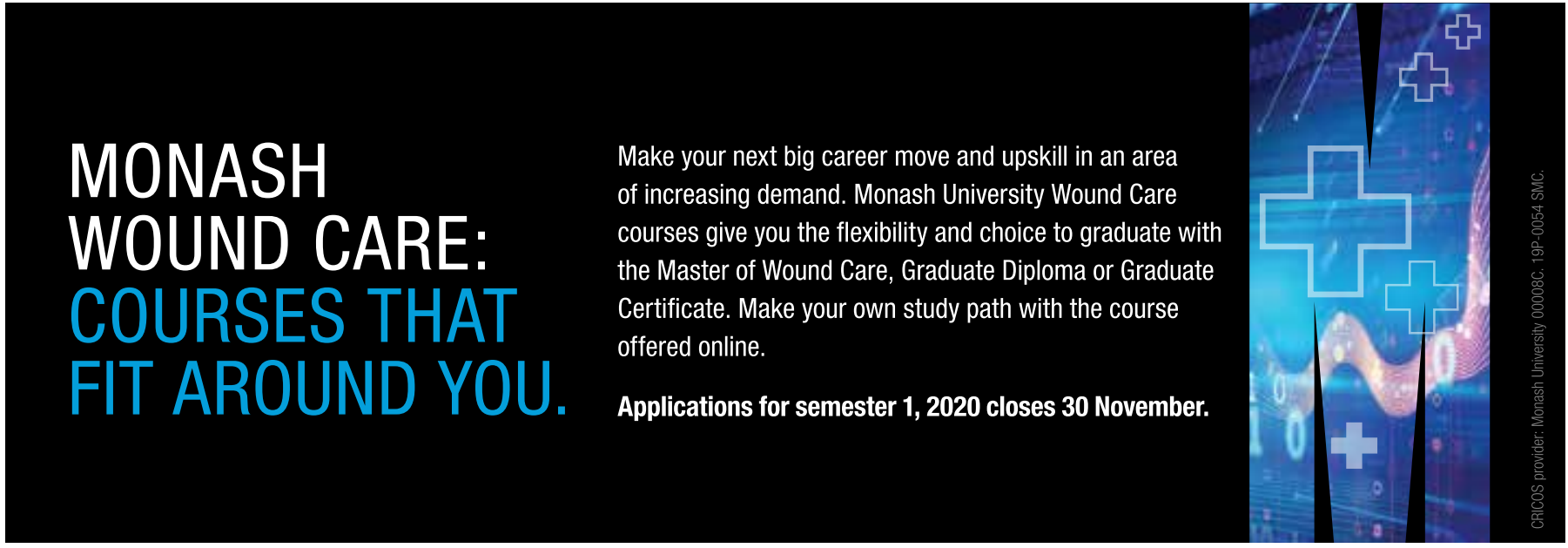

